\title{
Computational Aspects of Speed-Dependent Voigt Profiles
}

\author{
Franz Schreier \\ DLR - German Aerospace Center, Remote Sensing Technology Institute, \\ Oberpfaffenhofen, 82234 Weßling, Germany
}

\begin{abstract}
The increasing quality of atmospheric spectroscopy observations has indicated the limitations of the Voigt profile routinely used for line-by-line modeling, and physical processes beyond pressure and Doppler broadening have to be considered. The speed-dependent Voigt (SDV) profile can be readily computed as the difference of the real part of two complex error functions (i.e. Voigt functions). Using a highly accurate code as a reference, various implementations of the SDV function based on Humlíček's rational approximations are examined for typical speed dependences of pressure broadening and the range of wavenumber distances and Lorentz to Doppler width ratios encountered in infrared applications. Neither of these implementations appears to be optimal, and a new algorithm based on a combination of the Humlíček and Weideman (1994) rational approximations is suggested.
\end{abstract}

Keywords: Complex error function; Voigt function; Hartmann-Tran Lineshape; Humlicek-Weideman rational approximations

doi: http://dx.doi.org/10.1016/j.jqsrt.2016.08.009

\section{Introduction}

For a long time the Voigt profile 1] accounting for pressure/collision and Doppler broadening has been the standard for line-by-line modeling of infrared and microwave radiative transfer in the context of atmospheric remote sensing. Inadequacies of this profile have been observed since decades in molecular laboratory spectroscopy. Changes of molecular velocity due to collisions reduce the Doppler broadening, and several profiles have been developed for collisional (or Dicke) narrowing [2]. Moreover, the speed-dependence of the relaxation rates alters the Lorentz line shape describing the collision broadening [3, 4].

\footnotetext{
* Corresponding author

Email address: franz.schreier@dlr.de (Franz Schreier)
}

Preprint submitted to J. Quant. Spectr. \& Rad. Transfer July 22, accepted August 12, 2016 
In recent years, discrepancies between model and observation have also become evident in atmospheric spectroscopy. Schneider et al. [5, 6] and Boone et al. [7, 8] reported on improved retrievals with smaller residuals in the analysis of ground-based Fourier transform infrared solar absorption spectra and Atmospheric Chemistry Experiment - Fourier Transform Spectrometer (ACEFTS, Bernath et al. 9]) data, respectively, with speed-dependent Voigt profiles. Furthermore, non-Voigt lineshapes have also become an issue for (exo-) planetary atmospheric studies [10, 11]. In a recent IUPAC Technical Report [4 the partially Correlated quadratic-Speed-Dependent Hard-Collision profile (pCqSDHCP) has been recommended as the appropriate model for high resolution spectroscopy. The model, originally developed by Tran et al. 12 and therefore called Hartmann-Tran (or HT) profile for short, accounts for both collisional narrowing and speed-dependence.

From a computational point, both the SDV profile and the HT profile can be calculated readily from the complex error function (whose real part is the Voigt function). The SDV profile is essentially given by the difference of two Voigt profiles, and the HT profile also involves the difference of two complex error functions scaled with the square of their arguments. In view of these differences, highly accurate numerical algorithms are required for the computation of the convolution integral defining the Voigt and complex error functions, that do not have a closed-form analytical solution. Boone, Walker, and Bernath [8] (henceforth "BWB") suggest to use the Humlíček [13] w4 rational approximation (with a claimed accuracy of $10^{-4}$ ) for the SDV profile, and Tran et al. [12] recommend the slightly more accurate Humlíček 14 cpf12 rational approximation (six significant digits). Similar to most other complex error function codes, these two codes use different rational approximations in different regions of the complex plane (four regions in case of w4, two regions in cpf12). Accordingly, for the calculation of the SDV and HT profiles care has to be taken to ensure that both terms of the difference use the same approximation.

In this note we provide an assessment of these approximations for the speeddependent Voigt profile. After a brief review of the definitions and algorithms in the following section, we present comparisons against a reference code in section 3 and introduce an alternative implementation utilizing the Humlíček and Weideman [15] rational approximations. A summary and our conclusions are given in the final section 4. For simplicity, pressure induced line shift will be ignored. Python modules of the speed-dependent Voigt function along with rational approximations for the complex error function are provided in supplemental files. 


\section{Theory}

\section{1. "Standard" Voigt}

The Voigt profile $g_{\mathrm{V}}$ (units of reciprocal wavenumbers and normalized to one) and the dimensionless Voigt function $K$ (normalized to $\sqrt{\pi}$ ) are defined by

$$
\begin{aligned}
g_{\mathrm{V}}\left(\nu ; \hat{\nu}, \gamma_{\mathrm{L}}, \gamma_{\mathrm{D}}\right) & =\frac{\sqrt{\ln 2 / \pi}}{\gamma_{\mathrm{D}}} K(x, y) \\
K(x, y) & =\frac{y}{\pi} \int_{-\infty}^{\infty} \frac{\mathrm{e}^{-t^{2}}}{(x-t)^{2}+y^{2}} \mathrm{~d} t
\end{aligned}
$$

with the complex error function [16, 17, 18,

$$
\begin{aligned}
w(z) & =\frac{\mathrm{i}}{\pi} \int_{-\infty}^{\infty} \frac{\mathrm{e}^{-t^{2}}}{z-t} \mathrm{~d} t \\
& =K(x, y)+\mathrm{i} L(x, y) \quad \text { with } z=x+\mathrm{i} y
\end{aligned}
$$

where $x \equiv \sqrt{\ln 2}(\nu-\hat{\nu}) / \gamma_{\mathrm{D}}$ is a measure of the distance to the line center at wavenumber $\hat{\nu}$, and $y \equiv \sqrt{\ln 2} \gamma_{\mathrm{L}} / \gamma_{\mathrm{D}}$ is essentially the ratio of the Lorentzian and Doppler widths. Note that both $x$ and $y$ are dimensionless.

Numerous algorithms have been developed for the Voigt and complex error function exploiting a wide variety of numerical techniques. A survey of rational approximations [19, 14, 13, 15] has been given in Schreier [20, and a combination of the Humlíček 13] $R_{1,2}$ rational approximation (where the subscript denotes the degree of the numerator and denominator polynomials) and the Weideman [15] approximation has been recommended (called hum1wei32 henceforth),

$$
w(z)= \begin{cases}R_{1,2} \equiv \frac{\mathrm{i} z / \sqrt{\pi}}{z^{2}-1 / 2} & |x|+y>15 \\ \frac{\pi^{-1 / 2}}{L-\mathrm{i} z}+\frac{2}{(L-\mathrm{i} z)^{2}} \sum_{n=0}^{N-1} a_{n+1} Z^{n} & \text { otherwise }\end{cases}
$$

where the $a_{1}, \ldots, a_{N}$ are real-valued polynomial coefficients, $Z=\frac{L+\mathrm{i} z}{L-\mathrm{i} z}$ and $L=$ $2^{-1 / 4} N^{1 / 2}$. For $N=32$ the relative error $\Delta K / K_{\text {ref }}$ with $\Delta K(x, y) \equiv \mid K(x, y)-$ $K_{\text {ref }}(x, y) \mid$ is less than $8 \cdot 10^{-5}$ for $0 \leq x \leq 20$ and $10^{-6} \leq y \leq 10^{2}$ (with the largest error for $y=10^{-6}$, see [20, Fig. 8]).

\subsection{Speed-dependent Voigt}

According to Boone et al. 8 ] the speed-dependent Voigt profile can be computed as the difference of two complex error functions

$$
\begin{aligned}
g_{\mathrm{sdv}}\left(\nu ; \hat{\nu}, \gamma_{\mathrm{L}}, \gamma_{2}, \gamma_{\mathrm{D}}\right) & =\frac{\sqrt{\ln 2 / \pi}}{\gamma_{\mathrm{D}}} Q(\alpha, \beta, \delta) \\
Q(\alpha, \beta, \delta) & =\operatorname{Re}\left(w\left(\mathrm{i} z_{-}\right)-w\left(\mathrm{i} z_{+}\right)\right)
\end{aligned}
$$


where $\gamma_{2}$ is the quadratic term characterizing the speed dependence of the pressure broadening, and the "SDV function" $Q$ is defined in analogy with the Voigt function $K$ with a normalization $\int Q(\alpha, \beta, \delta) \mathrm{d} \beta=2 \sqrt{\pi \delta}$. The complex argument $\mathrm{i} z_{ \pm}$

$$
\begin{aligned}
z_{ \pm}= & \sqrt{\alpha+\delta+\mathrm{i} \beta} \pm \sqrt{\delta} \\
= & \pm \sqrt{\delta}+\frac{1}{\sqrt{2}} \sqrt{\sqrt{(\alpha+\delta)^{2}+\beta^{2}}+\alpha+\delta} \\
& +\operatorname{sign}(\beta) \frac{\mathrm{i}}{\sqrt{2}} \sqrt{\sqrt{(\alpha+\delta)^{2}+\beta^{2}}-\alpha-\delta} \\
= & \pm \sqrt{\delta}+\frac{1}{\sqrt{2} \sqrt{\sqrt{(\alpha+\delta)^{2}+\beta^{2}}+\alpha+\delta}} \\
& +\frac{\mathrm{i} \beta / \sqrt{2}}{\sqrt{\sqrt{(\alpha+\delta)^{2}+\beta^{2}}+\alpha+\delta}}
\end{aligned}
$$

depends on three variables ${ }^{1}$

$$
\begin{aligned}
\alpha & =\frac{\gamma_{\mathrm{L}}}{\gamma_{2}}-\frac{3}{2}=2 y \sqrt{\delta}-\frac{3}{2} \\
\beta & =\frac{\nu-\hat{\nu}}{\gamma_{2}}=2 x \sqrt{\delta} \\
\delta & =\frac{1}{4 \ln 2}\left(\frac{\gamma_{\mathrm{D}}}{\gamma_{2}}\right)^{2} .
\end{aligned}
$$

Note that the real part of the $w$ argument (i.e. the imaginary part of $z_{ \pm}$) is identical; furthermore, both the real and imaginary part of $z_{ \pm}$depend on the "wavenumber difference" $x \propto \beta$. The alternative form 10 might be computationally advantageous as it requires only two instead of three square root evaluations and avoids possible inaccuracies for $\beta^{2} \ll(\alpha+\delta)^{2}$. Furthermore there is no need to evaluate the sign function (Note that the numpy sign function returns $+1,-1$, and 0 for positive, negative, and zero argument consistent with (9), but the Fortran intrinsic is defined differently).

The HT profile requires one to compute the difference $z_{-}^{2} w\left(\mathrm{i} z_{-}\right)-z_{+}^{2} w\left(\mathrm{i} z_{+}\right)$ in addition, where the arguments $z_{ \pm}$also depend on two further parameters $\eta$ and $\nu_{\mathrm{vc}}$ (see Tennyson et al. 4 for details). If $\eta \rightarrow 0$, the HT profile reduces to a speed-dependent Rautian profile, essentially defined by $Q /\left(1-\nu_{\mathrm{vc}} Q\right)$; if both parameters vanish, the HT profile reduces to the SDV profile. Finally, if the speed-dependence parameter $\gamma_{2}$ vanishes, the SDV profile reduces to the Voigt profile.

\footnotetext{
${ }^{1} \mathrm{HT}$ [4, 12] notation: $Y \equiv \delta$ and $X \equiv \mathrm{i} \frac{\nu-\hat{\nu}}{\gamma_{2}}+\frac{\gamma_{\mathrm{L}}}{\gamma_{2}}-\frac{3}{2}=\mathrm{i} \beta+\alpha$ and $Z_{ \pm}=\sqrt{X+Y} \pm \sqrt{Y}$. The function $\operatorname{Re}(A)$ defined in Eq. (7) of Tennyson et al. 4 is identical to the "SDV function" $Q$ except for a factor $\sqrt{\pi \ln 2} / \gamma_{\mathrm{D}}$. Note that HT uses capital $\Gamma$ instead of the lower case $\gamma$. Furthermore note that in Eq. (11) of [12] the Im operator is missing, i.e. the asymptotic limit for small $\delta$ is $Q(\alpha, \beta, \gamma) \approx 4 \sqrt{\delta}\left[\frac{1}{\sqrt{\pi}}-\operatorname{Im}(\sqrt{\alpha+\mathrm{i} \beta} w(\mathrm{i} \sqrt{\alpha+\mathrm{i} \beta}))\right]$.
} 

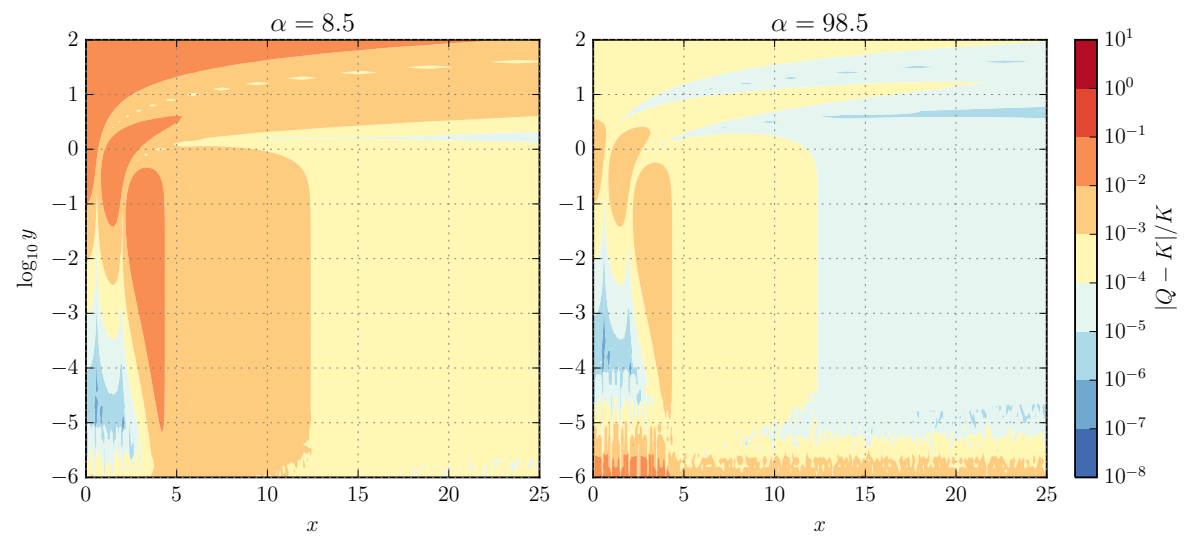

Figure 1: Contour plot of relative differences of the SDV function for $\alpha=8.5$ and $\alpha=98.5$ to the Voigt function.

\section{Results}

Because the SDV function in (7) depends on three variables $\alpha, \beta$, and $\delta$, visualization of differences with contour plots similar to 20] is difficult. However, according to $11 \mathrm{~b}, \beta$ is essentially a scaled equivalent of the standard Voigt parameter $x$, and $\sqrt{\delta}=(\alpha+3 / 2) /(2 y)$. Moreover, because the pressure and temperature dependence of $\gamma_{\mathrm{L}}$ and $\gamma_{2}$ is often assumed to be identical, i.e. $\gamma_{\mathrm{L}, 2} \sim p / T^{n}$, contour plots of the relative difference will be shown with the $x$ and $y$ axes. Two cases will be explored for $\gamma_{\mathrm{L}} / \gamma_{2}=10$ (or $\alpha=8.5$ ) and $\gamma_{\mathrm{L}} / \gamma_{2}=100(\alpha=98.5)$ (see e.g. Tables 1 and 2 of Rohart et al. [21] or Table 3 of Schneider et al. [6]); $\alpha=8.5$ is also close to the "default" implemented in the BWB code. These cases do not cover all relevant situations, but they might help to reveal possible problems of the implementations.

\subsection{The SDV function compared to the Voigt function}

To become more familiar with the impact of speed dependence, we show the relative difference of the SDV function to the Voigt function in Fig. 1. Both functions were computed with the wof $z$ algorithm, a combination of the Poppe and Wijers 22, 23] and Zaghloul and Ali 24] approximations with a stated accuracy of at least 13 significant digits (Scientific Python scipy . special . wofz implementation, see also http://ab-initio.mit.edu/Faddeeva). The relative difference $(Q-K) / K$ reaches almost 0.04 for $\alpha=8.5$ (at $x=1.5$ and $y=0.398$ ), and 0.17 for $\alpha=98.5$ (at $x=0.4$ and $y=10^{-6}$ ). For intermediate $y$, the largest differences appear for intermediate $x$ and decrease towards the center and in the wings. Fig. 2 displays the $Q$ function for $y=0.398$ and the two $\alpha$ 's and their relative deviation to the Voigt function.

\subsection{Range of independent variables}

Before assessing the applicability of the various complex error function algorithms, it is useful to check the range of the $\mathrm{i} z_{ \pm}$arguments. For Earth atmo- 


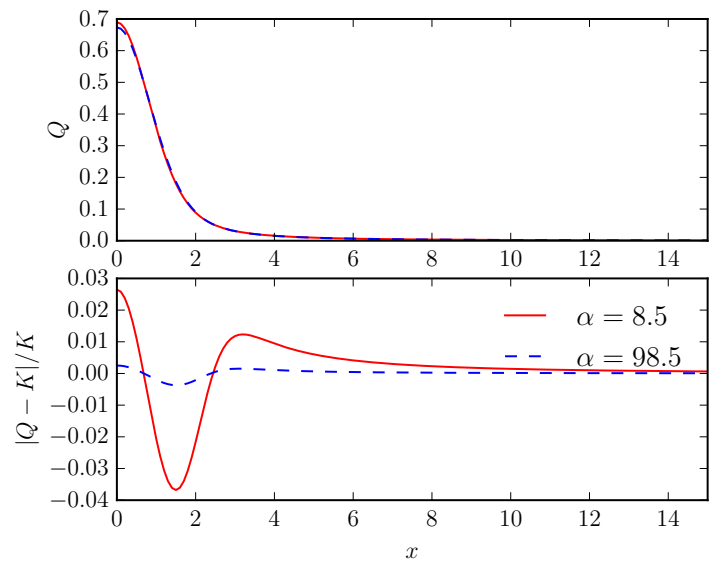

Figure 2: The SDV function for $y=0.398$ with $\alpha=8.5$ and $\alpha=98.5$ (top) and their relative difference to the Voigt function $K$ (bottom). The second plot essentially represents a cut of the contour plot 1 at $y=0.398$.

spheric spectroscopy the Lorentz to Doppler width ratio varies over 10 orders of magnitude, $10^{-6} \leq y \leq 10^{4}$, and even higher $y$ values are found in microwave spectroscopy of the Venusian atmosphere [20, Figures 2b and 3b]. For thermal infrared observations such as provided by ground-based or spaceborne spectrometers $y$ values up to about 100 are relevant.

Fig. 3 shows that the imaginary part of the arguments $\mathrm{i} z_{ \pm}$spans an even larger range of values up to $10^{10}$, whereas the real part does not change dramatically compared to the Voigt function variable $x$.

\subsection{Assessment of the Humliček rational approximations}

In the Fortran code provided as supplement of the BWB paper, the SDV profile is computed using the Humlíček [13] w4 code for $w(z)$ that utilizes different rational approximations for large, intermediate, and small $|x|+y$ (the inner region is further split in two subregions). For evaluation of the difference of $w\left(\mathrm{i} z_{+}\right)$and $w\left(\mathrm{i} z_{-}\right)$BWB compute the two terms using the rational approximation appropriate for the smaller of the two arguments. This approach is clearly only justified, if the approximation used in an inner region is also applicable to the outer region. It is tempting to assume that higher order rational approximations (as required for a smaller argument $z$ ) are also valid for large $z$. In Fig. 4 we show the relative accuracy of the individual rational approximations (used for the entire $x, y$ plane) with respect to the wof $z$ algorithm. Clearly, this assumption is confirmed for the $R_{3,4}$ approximation originally designed for the $5.5 \leq|x|+y<15$ region that can also be used in the outer region. However, using one of the higher order rational approximations outside their intended region can be quite dangerous.

Fig. 5 demonstrates that in case of the older cpf 12 algorithm the approximation for region II $(y<0.85$ and $|x|>18.1 y+1.65)$ can also be used for moderately large $y$ up to 100 , whereas the region I approximation cannot be used for small $y$ and large $|x|$. However, in view of Fig. 3 it is important to recognize that the region II approximation completely fails for large $y$. 

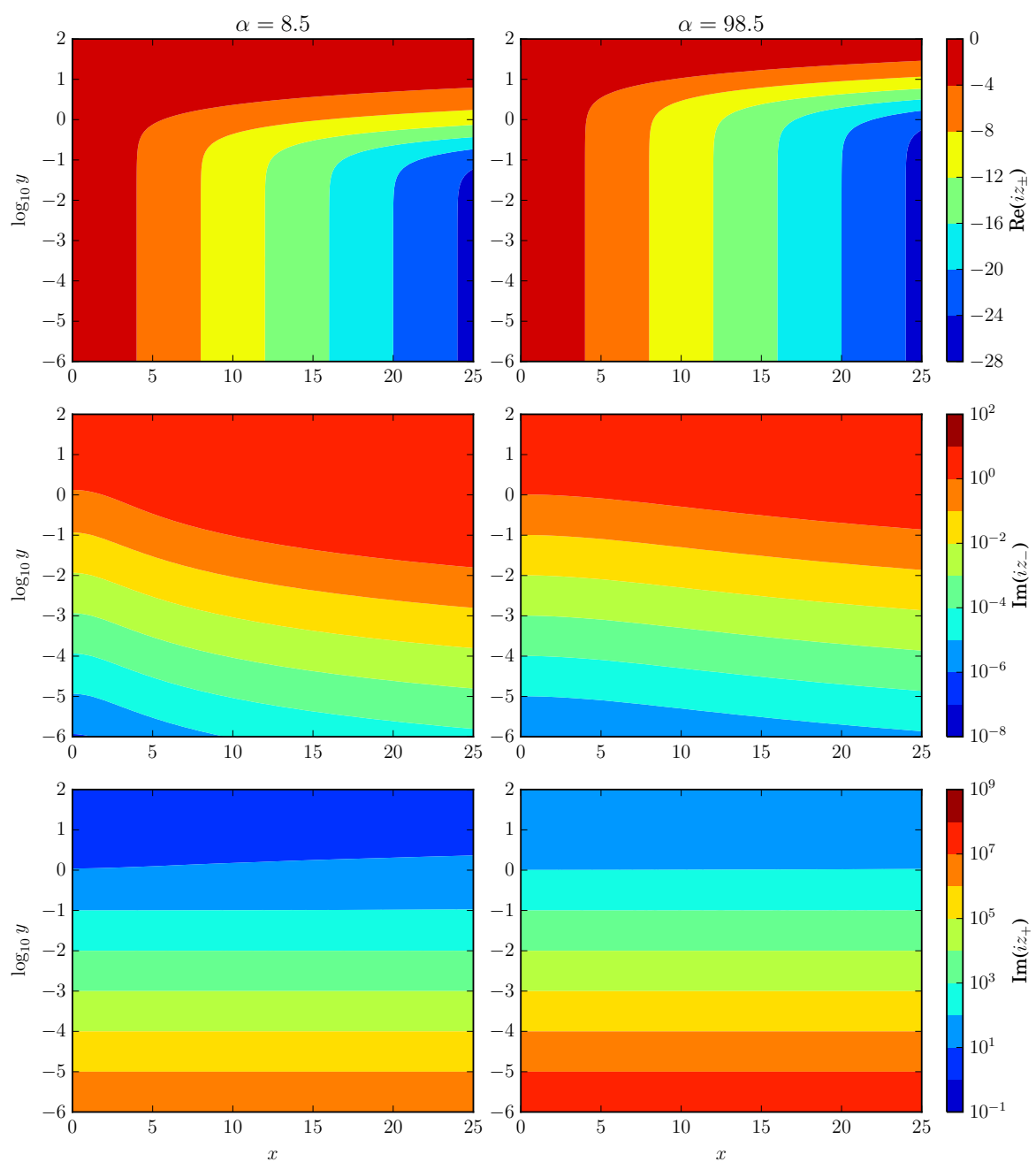

Figure 3: The real and imaginary part of the arguments $\mathrm{i} z_{ \pm}$of the two complex error functions in (7) for $\gamma_{\mathrm{L}} / \gamma_{2}=10$ (left) and $\gamma_{\mathrm{L}} / \gamma_{2}=100$ (right). Note the different color bars. 

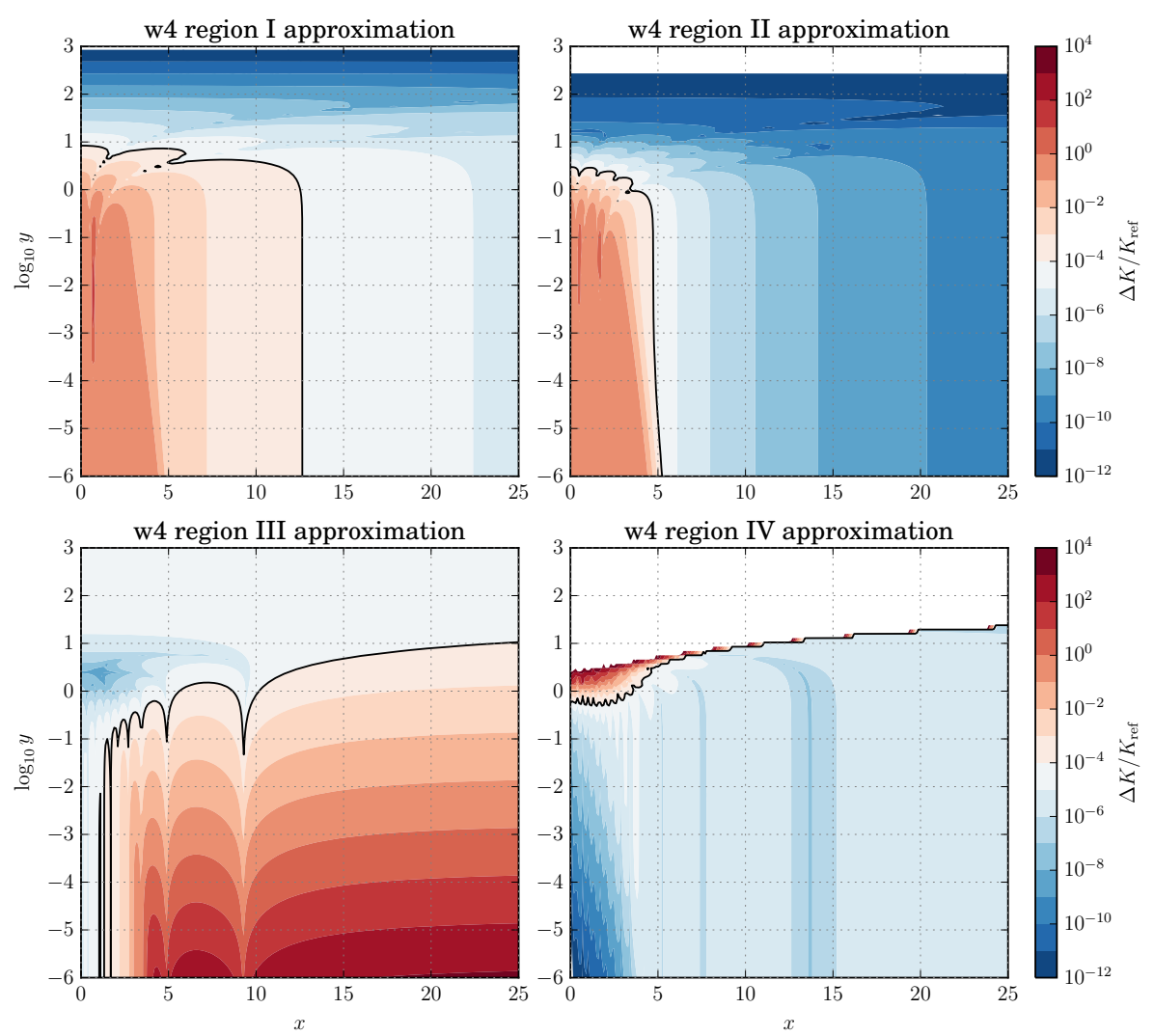

Figure 4: Relative accuracy of the four rational approximations used in the Humlíček [13] w4 code with $K_{\text {ref }}$ given by the wofz code. (White areas correspond to deviations less than $10^{-12}$, the black line indicates the $10^{-4}$ contour.)

\section{4. "Naive" implementations of the SDV function}

In a first implementation of the SDV function, the two terms $w\left(\mathrm{i} z_{ \pm}\right)$are evaluated independently using either of the Humliček approximations. The top row of Fig. 6 clearly shows the problems of the Humlíček [13] w4 approximation for large $x$ and small $y$. The largest error occurs at $z=13.5+0.158 \mathrm{i}$ (see Tab. 1); both $\mathrm{i} z_{ \pm}$are large, so both terms $w\left(\mathrm{i} z_{ \pm}\right)$are computed using the $R_{1,2}$ approximation appropriate for the outer region I. However, despite the four significant digits of $\mathrm{w} 4$, the difference appears to be problematic, i.e. the w4-SDV and the wofz-SDV agree on two significant digits only. This obvious failure of the $R_{1,2}$ approximation is confirmed by using the intermediate region II approximation $R_{3,4}$ also in the outer region I $(|x|+y>15$, Fig. 4 indicates an accuracy better than $10^{-8}$ in this region) that significantly reduces the relative differences in the lower right part of the contour plots.

Tab. 1 also gives details for the failure around $x=4.2$ and $y=0.25$, where w4-SDV and wofz-SDV agree on three digits. In this case, i $z_{-}$lies in region III, 

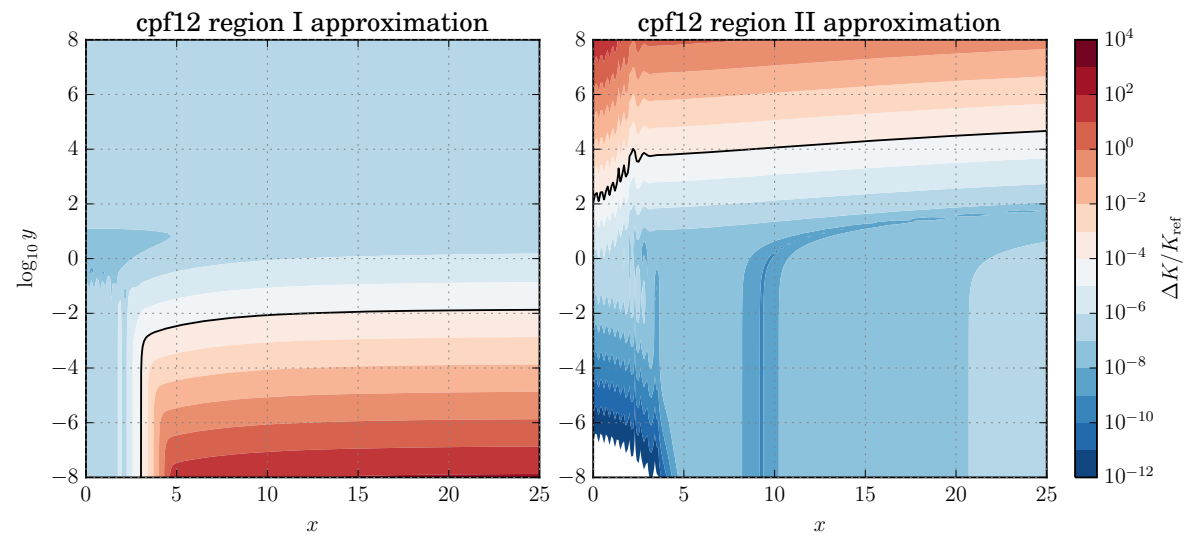

Figure 5: Relative accuracy of the two 12-term rational approximations used in the cpf 12 code with $K_{\text {ref }}$ given by the wofz code. Left: Eq. (6), Right: Eq. (11) of Humlíček [14]

\begin{tabular}{rccc} 
& w4 & cpf12 & wofz \\
\hline $\mathrm{i} z_{-}=-12.505+2.5127 \mathrm{i}$ & 0.00879149 & 0.00879221 & 0.00879221 \\
$\mathrm{i} z_{+}=-12.504+65.608 \mathrm{i}$ & 0.00829714 & 0.00829714 & 0.00829714 \\
$z=+13.5+0.1585 \mathrm{i}$ & 0.00049435 & 0.00049507 & 0.00049507 \\
\hline & & & \\
$\mathrm{i} z_{-}=-4.0730+0.6205 \mathrm{i}$ & 0.02269985 & 0.02269767 & 0.02269768 \\
$\mathrm{i} z_{+}=-4.0730+40.431 \mathrm{i}$ & 0.01381008 & 0.01381008 & 0.01381009 \\
$z=+4.2+0.2512 \mathrm{i}$ & 0.00888976 & 0.00888759 & 0.00888760 \\
\hline
\end{tabular}

Table 1: Examination of some large differences in the top-left of Fig. 6 sdv_w4 vs. sdv_cpf 12 and $\mathbf{s d v}_{-}$wof $z$ for $\alpha=8.5$. The first two rows of each block give the $\operatorname{Re}(w)$ function values for $\mathrm{i} z_{ \pm}$, the third row the difference, i.e. the $Q$ function value.

whereas $\mathrm{i} z_{+}$is in region I. For both cases the table also gives the Voigt function values obtained from the $\mathrm{cpf} 12$ routine for comparison.

The Humlíček [14] approximation cpf12 (used in the codes provided as supplement of the Tran et al. [12 paper) gives SDV function values in good agreement with the wofz code (Fig. 6 bottom). This essentially confirms the numerical tests of Tran et al. [12, who found relative errors up to $10^{-4}$ for the HT profile using the cpf 12 subroutine compared to a reference based on direct numerical integration. Note that Tran et al. [12] use a "slightly improved" cpf12 subroutine with a 15-term asymptotic expansion (http://dlmf .nist.gov/7.12.E1 or Eq. (7.1.23) in Abramowitz and Stegun [16]) for $\sqrt{x^{2}+y^{2}}>8$. In the subroutine provided as supplement, Tran et al. [12] ensure that both terms $w\left(\mathrm{i} z_{ \pm}\right)$are computed either with the original $\operatorname{cpf} 12$ routine (with the appropriate rational approximation for $\mathrm{i} z_{+}$and $\mathrm{i} z_{-}$selected independently) or the asymptotic approximation. 

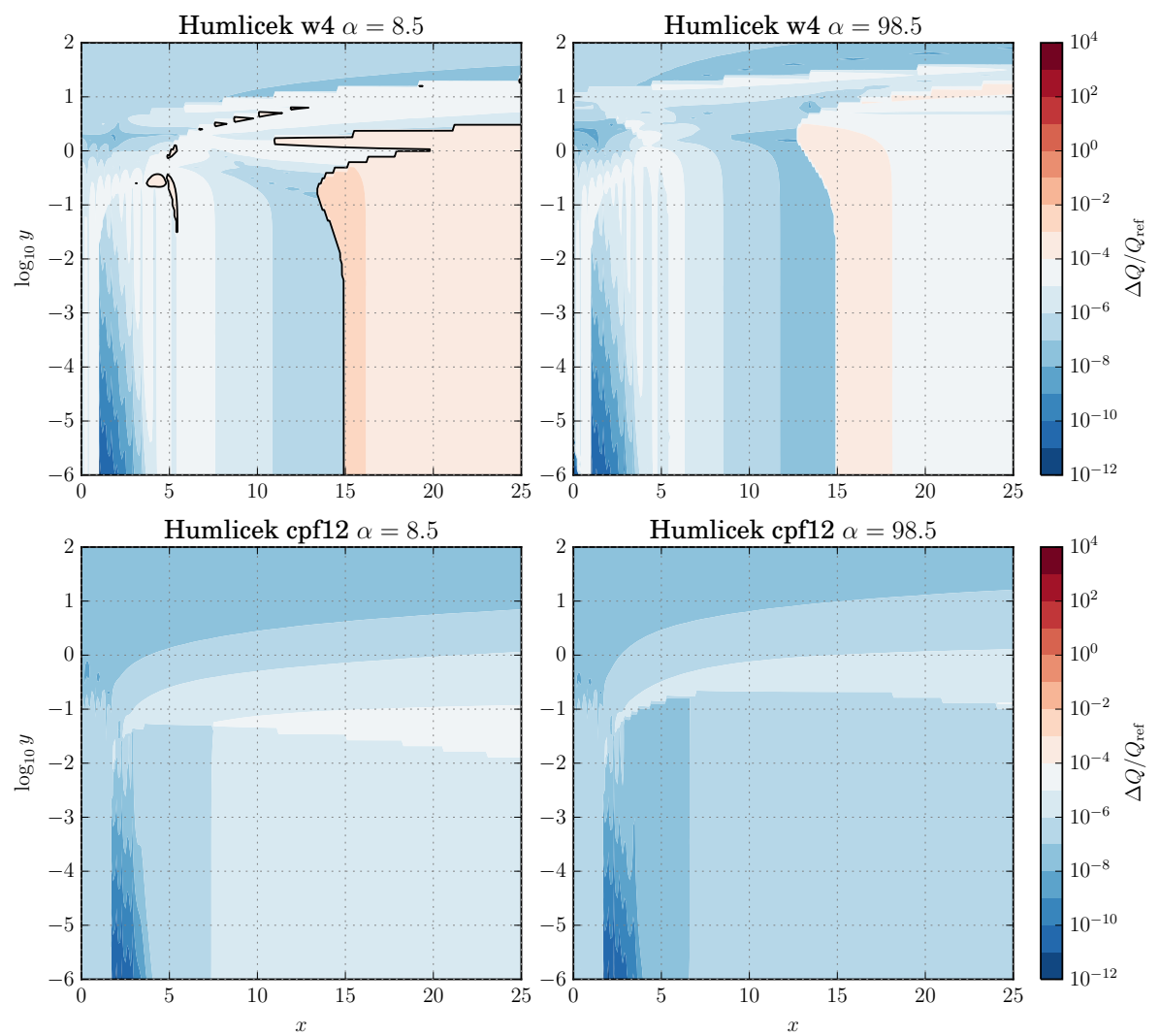

Figure 6: Relative accuracy of the "naive" implementations of the SDV function using w4 (top) and $\operatorname{cpf} 12$ (bottom) for the complex error function. The maximum deviations are $1.45 \cdot 10^{-3}$ and $2.14 \cdot 10^{-4}$ for $\mathrm{w} 4$, and $8.18 \cdot 10^{-5}$ and $1.05 \cdot 10^{-5}$ for $\mathrm{cpf} 12$.

\subsection{A consistent use of the Humlícek w4 approximations for the SDV function}

As emphasized by Boone et al. 8, and Tran et al. [12, evaluation of the difference might suffer from accuracy problems, when the two Voigt functions are computed using different approximations. However, using a more sophisticated SDV routine based on the Humlíček [13] w4 code with both arguments $\mathrm{i} z_{ \pm}$evaluated consistently with the same approximation reveals several problems. First, Fig. 6 already demonstrated that the difference of the two $R_{1,2}$ approximations can fail for large $x$ and small $y$. Secondly, for $|x|<5.5$ and small $y$ both terms will be evaluated using the region IV approximation, a $R_{13,14}$ rational approximation ${ }^{2}$ with an additional exponential term.

\footnotetext{
${ }^{2}$ The rational approximation for region IV has been incorrectly termed $R_{6,7}$ in Eq. (25) of Schreier [20, i.e. the quotient of two polynomials of degree six and seven. Actually the denominator polynomial is a seven-degree polynomial in $z^{2}$, and the numerator is a six-degree polynomial in $z^{2}$ multiplied with $z$.
} 

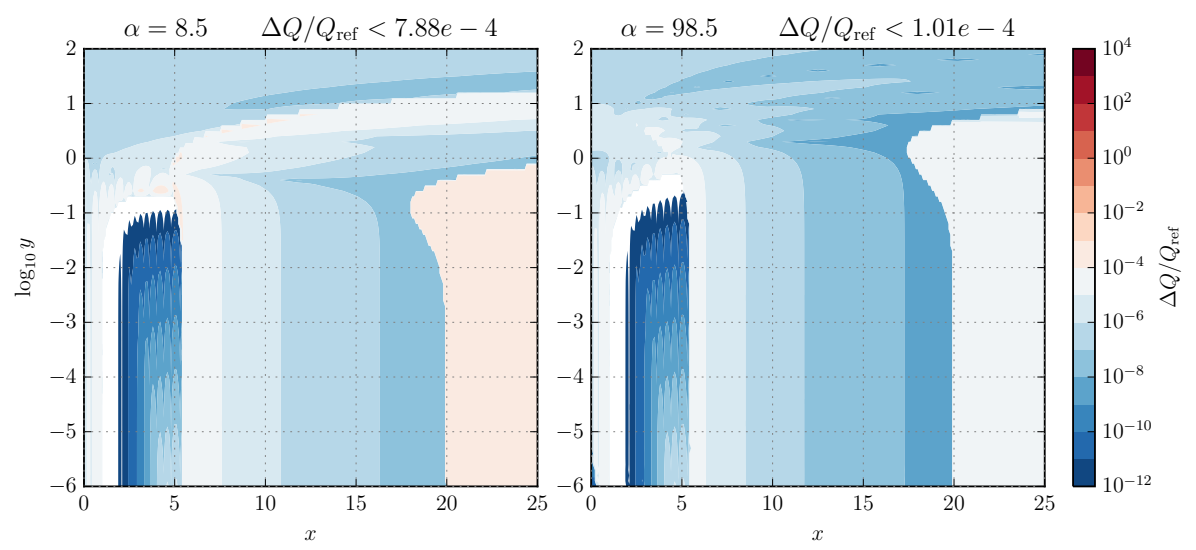

Figure 7: Relative accuracy of Humlíček w4 (with Weideman approximation for region IV) for the SDV function. Same approximation for $\mathrm{i} z_{+}$and $\mathrm{i} z_{-}$consistently.

Whereas $z_{-}$is small (hence the region IV approximation), $\operatorname{Im}\left(z_{+}\right)$can be very large (see Fig. 3), and Python/NumPy reports RuntimeWarning: overflow encountered in exp and the SDV function values returned are inf.

To circumvent these problems, the bound for region I has been moved outwards to $s=|x|+y=20$ and the region IV approximation has been replaced by the Weideman 32 term approximation. However, Fig. 7 clearly indicates that despite these changes significant deviations to the reference happen for especially large $x$ and small $y$.

\subsection{The Humliček-Weideman combination}

Although the Humlíček [14] cpf12 rational approximation provides accurate SDV functions (when validated against the wof $z$ code), use of this code appears to be suboptimal from an efficiency point of view. In its original implementation (essentially used in the Tran et al. [12] code), 12 divisions per function value are performed in region I, and 24 divisions and the evaluation of an exponential are required in region II. Furthermore, a (DO) loop to sum the individual quotients [14, Eq. (6)] or [20, Eq. (24)] is executed for each function value, and the routine is used in a scalar fashion.

Though it is possible to accelerate the code by vectorization [25] and by rewriting the sum of quotients to a single rational expression (straightforward for region I, cf. [20, subsection 5.2]), the w4 code or the Humlíček-Weideman combination are considerably faster [see 20, Fig. 9]. Furthermore, a SDV routine using only two regions is significantly easier to implement.

Because of the problems of the Humlíček 13] $R_{1,2}$ rational approximation discussed above (cf. Fig. 6] and 7), the Weideman [15] rational approximation (with $N=32$ ) and the Humlíček rational approximation

$$
R_{3,4} \equiv \frac{\mathrm{i} z\left(z^{2} / \sqrt{\pi}-1.410474\right)}{\left(z^{2}-3\right) z^{2}+3 / 4}
$$



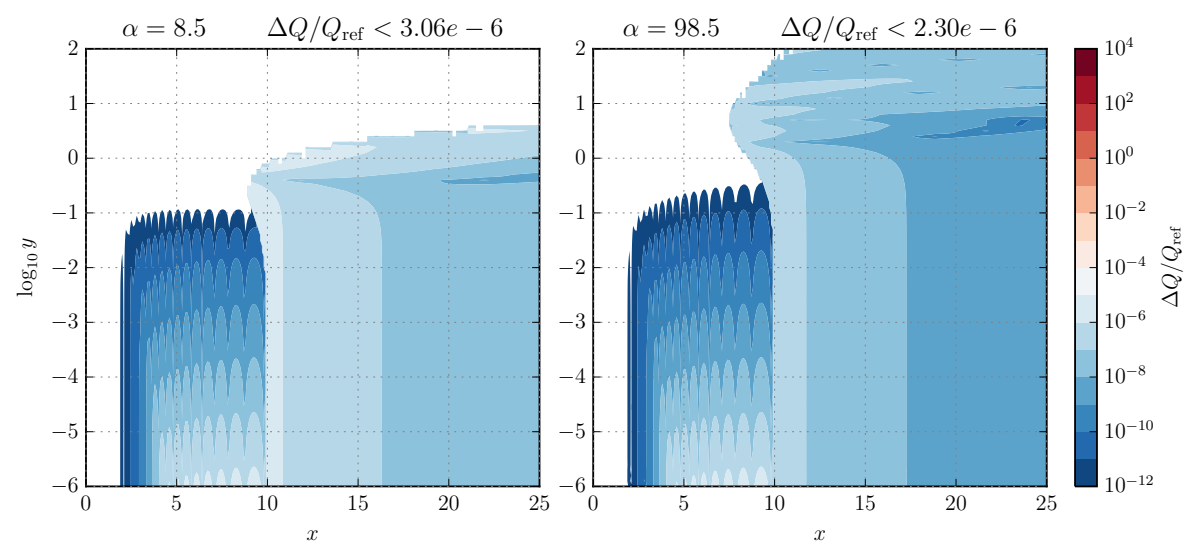

Figure 8: Relative accuracy of a consistent SDV function implementation based on the Humlíček and Weideman approximations (sdv hum2wei32c).

are used to evaluate the SDV function in a consistent way, i.e. using the Weideman approximation whenever one of the arguments is small $\left(s_{ \pm} \equiv\left|x_{ \pm}\right|+y_{ \pm}<\right.$ 10). In Fig. 8 the relative accuracy is shown, indicating small errors up to $3 \cdot 10^{-6}$. As an alternative, the Weideman [15] approximation could be combined with the Humlíček $R_{1,2}$ approximation (as in Schreier [20]), but in view of Fig. 7 the switch to the asymptotic approximation has to be shifted to $s=50$ or more. A distinct advantage is that the Weideman algorithm is valid in the entire $x, y$ plane; therefore, if one of the two arguments $\mathrm{i} z_{ \pm}$is near the origin and the other is in the asymptotic region, then both $w\left(\mathrm{i} z_{ \pm}\right)$can be computed safely with the Weideman approximation.

The Humlíček $R_{3,4}$ - Weideman combination ("hum2wei32") with a cut at $s=8$ provides Voigt function values as good as the cpf 12 rational approximation (maximum relative deviations to wofz $2 \cdot 10^{-6}$ ). In view of Fig. 6 (bottom) it is therefore tempting to use it in the "naive" implementation (called sdv_hum2wei32n in contrast to sdv_hum2wei32c discussed in the previous paragraph) for the SDV function. Fig. 9 confirms that with such an accurate complex error function code the two terms $w\left(\mathrm{i} z_{ \pm}\right)$can be computed independently.

\subsection{Efficiency}

First note that the Humlíček $R_{3,4}$ approximation, like $R_{1,2}$, requires one division, so the computational work is only slightly higher because of the doubled number of multiplications. Secondly, the effort to evaluate the Weideman approximation is higher compared to $R_{1,2}$ or $R_{3,4}$, however, this does not have a big impact on the overall computing time as the majority of function evaluations are performed in the line wings. Note, however, that many lbl codes use some kind of "multigrid" approach to compute function values in the line wings more efficiently [e.g. 26, 27, 28, 29, 30].

To quantify these expectations we first tested the various complex error function codes using IPython's builtin "magic" \%timeit (see http://ipython. 

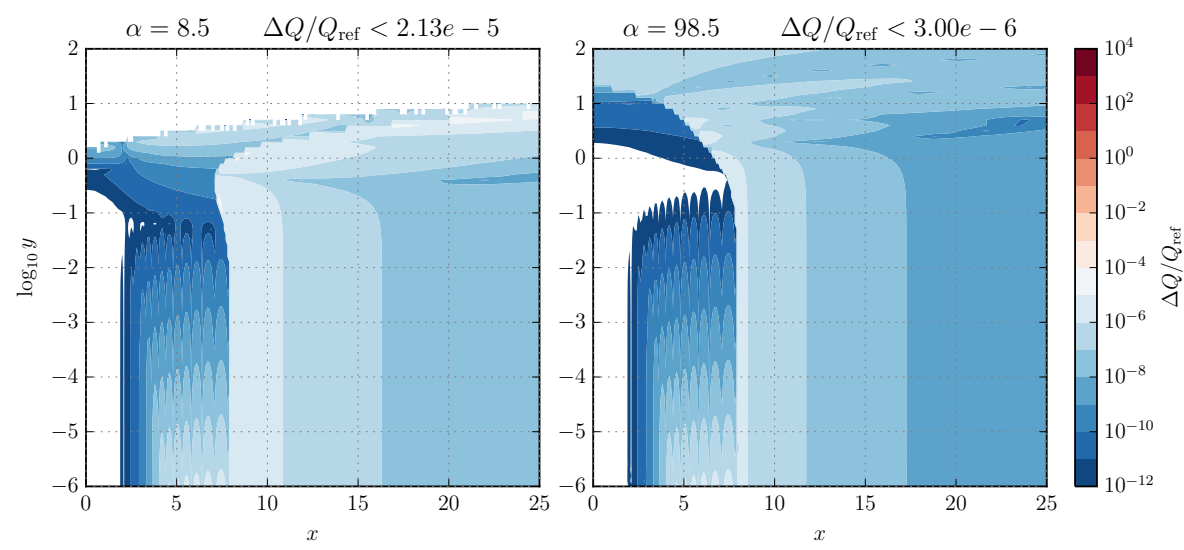

Figure 9: Relative accuracy of a "naive" implementation of the SDV function (sdv_hum2wei32n) using the Humlíček-Weideman combination hum2wei32.

org). Calling the Humlíček-Weideman combinations with the "matrix" of $x$ and $y$ used to generate the contour plots above indicates that hum2wei32 and hum1wei32 have roughly the same speed, and cpf 12 and w4 are about a factor 3 and 7 slower, respectively. For $\alpha=8.5$, the "naive" SDV function implementation using hum2wei32 is almost a factor five faster than the implementation using cpf 12 .

For operational data processing compiled languages are mandatory. Hence we have implemented the SDV function in Fortran 2003 and tested its efficiency in a way similar to the benchmarks reported in [20]. Fig. 10 shows that the Weidemann 32-term and Humlíček]s cpf12 approximation are considereably slower than the "naive" sdv_hum2wei32n code or the "consistent" implementation sdv_hum2wei32c. The "naive" implementation based on Humlíčeks w4 routine is as fast as sdv_hum2wei32n, but not recommended because of the accuracy problems (see Fig. 6).

\section{Summary and Conclusions}

Various implementations of the SDV function based on the Humlíček 13, 14 rational approximations for the complex error function have been surveyed and their relative accuracy with respect to a reference using the highly accurate wofz code has been studied. For the range of $y$ values (Lorentz to Doppler width ratio) typical for thermal infrared atmospheric spectroscopy applications and two representative values of $\gamma_{\mathrm{L}} / \gamma_{2}$, the SDV routine using the w4 code with four significant digits reveals accuracy problems especially for large $x$ and small $y$. The cpf 12 code with six digits accuracy provides SDV function values with better agreement to the reference (errors less than $10^{-5}$ ), but the efficiency of this code is significantly worse compared to w4. A new implementation utilizing a combination of the Weideman [15] 32-term rational approximation and the Humlíček [13] $R_{3,4}$ rational approximation (originally used in the w4 code for 


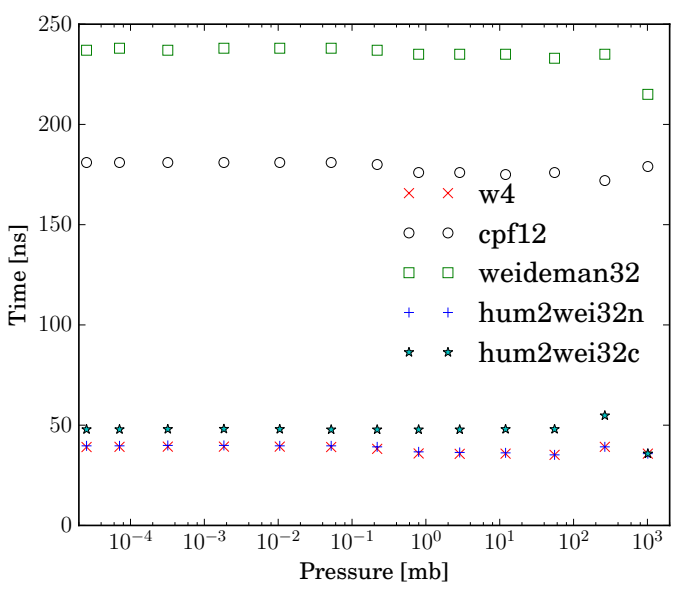

Figure 10: Time (in nanoseconds) per number of lines and number of function values (i.e., wavenumber grid points) for several implementations of the SDV function. The first four curves are based on the "naive" implementation. (The "sdv" prefix has been omitted in the legend.) Cross sections in the interval $16-17 \mathrm{~cm}^{-1}$ with $\alpha=8.5$ computed with the GNU gfortran compiler (version 4.8.1).

intermediate values of $z$ ) is suggested, that provides a relative accuracy of $10^{-5}$ or better in the entire $x, y$ plane.

In conclusion, a complex error function with more than four significant digits is recommended to provide the SDV function (i.e. Voigt function differences) with reasonable accuracy. Given an accurate algorithm, evaluation of the two terms of the difference using different approximations does not appear to be critical. However, if an algorithm uses the same approximation for both arguments, at least one of them should be applicable in the entire $x, y$ domain.

\section{Acknowledgments}

Thanks to Thomas Trautmann for critical reading of the manuscript and Philipp Hochstaffl for testing the Python scripts. Furthermore I would like to thank Sebastián Gimeno García, leaving the institute after more than ten years, for numerous helpful discussions. Finally I am grateful for the reviewer to suggest the alternative formulation 10 .

\section{References}

[1] B.H. Armstrong. Spectrum line profiles: The Voigt function. J. Quant. Spectrosc. \& Radiat. Transfer, 7:61-88, 1967. doi: 10.1016/0022-4073(67)90057-X.

[2] P.L. Varghese and R.K. Hanson. Collisional narrowing effects on spectral line shapes measured at high resolution. Appl. Opt., 23(14):2376-2385, 1984. doi: 10.1364/AO.23. 002376.

[3] J.M. Hartmann, C. Boulet, and D. Robert. Collisional Effects on Molecular Spectra. Elsevier, 2008.

[4] J. Tennyson, P.F. Bernath, A. Campargue, A.G. Császár, L. Daumont, R.R. Gamache, J.T. Hodges, D. Lisak, O.V. Naumenko, L.S. Rothman, H. Tran, N.F. Zobov, J. Buldyreva, C.D. Boone, M.D. De Vizia, L. Gianfrani, J.-M. Hartmann, R. McPheat, D. Weidmann, J. Murray, N.H. Ngo, and O.L. Polyansky. Recommended isolated-line profile for representing high-resolution spectroscopic transitions (IUPAC technical report). Pure Appl. Chem., 86(12):1931-1943, 2014. doi: 10.1515/pac-2014-0208. 
[5] M. Schneider and F. Hase. Improving spectroscopic line parameters by means of atmospheric spectra: Theory and example for water vapor and solar absorption spectra. J. Quant. Spectrosc. \& Radiat. Transfer, 110(17):1825 - 1839, 2009. doi: 10.1016/j.jqsrt.2009.04.011.

[6] M. Schneider, F. Hase, J.-F. Blavier, G.C. Toon, and T. Leblanc. An empirical study on the importance of a speed-dependent Voigt line shape model for tropospheric water vapor profile remote sensing. J. Quant. Spectrosc. \& Radiat. Transfer, 112(3):465-474, 2011. doi: $10.1016 /$ j.jqsrt.2010.09.008.

[7] C.D. Boone, K.A. Walker, and P.F. Bernath. Speed-dependent Voigt profile for water vapor in infrared remote sensing applications. J. Quant. Spectrosc. E Radiat. Transfer, 105:525-532, 2007. doi: 10.1016/j.jqsrt.2006.11.015.

[8] C.D. Boone, K.A. Walker, and P.F. Bernath. An efficient analytical approach for calculating line mixing in atmospheric remote sensing applications. J. Quant. Spectrosc. 83 Radiat. Transfer, 112(6):980 - 989, 2011. doi: 10.1016/j.jqsrt.2010.11.013.

[9] P. Bernath, C. T. McElroy, M. C. Abrams, C. D. Boone, M. Butler, C. Camy-Peyret, M. Carleer, C. Clerbaux, P.-F. Coheur, R. Colin, P. DeCola, M. DeMaziere, J. R. Drummond, D. Dufour, W. F. J. Evans, H. Fast, D. Fussen, K. Gilbert, D. E. Jennings, E. J. Llewellyn, R. P. Lowe, E. Mahieu, J. C. McConnell, M. McHugh, S. D. McLeod, R. Michaud, C. Midwinter, R. Nassar, F. Nichitiu, C. Nowlan, C. P. Rinsland, Y. J. Rochon, N. Rowlands, K. Semeniuk, P. Simon, R. Skelton, J. J. Sloan, M.-A. Soucy, K. Strong, P. Tremblay, D. Turnbull, K. A. Walker, I. Walkty, D. A. Wardle, V. Wehrle, R. Zander, and J. Zou. Atmospheric Chemistry Experiment (ACE): Mission overview. Geophys. Res. Letters, 32:L15S01, 2005. doi: 10.1029/2005GL022386.

[10] M. Snels, S. Stefani, D. Grassi, G. Piccioni, and A. Adriani. Carbon dioxide opacity of the Venus' atmosphere. Planet. Space Sci., 103:347 - 354, 2014. doi: 10.1016/j.pss.2014. 08.002 .

[11] J.J. Fortney, T.D. Robinson, S. Domagal-Goldman, D. Skålid Amundsen, M. Brogi, M. Claire, D. Crisp, E. Hebrard, H. Imanaka, R. de Kok, M.S. Marley, D. Teal, T. Barman, P. Bernath, A. Burrows, D. Charbonneau, R.S. Freedman, D. Gelino, C. Helling, K. Heng, A.G. Jensen, S. Kane, E.M.-R. Kempton, R.K. Kopparapu, N.K. Lewis, M. Lopez-Morales, J. Lyons, W. Lyra, V. Meadows, J. Moses, R. Pierrehumbert, O. Venot, S.X. Wang, and J.T. Wright. The need for laboratory work to aid in the understanding of exoplanetary atmospheres. ArXiv e-prints, (1602.06305), February 2016.

[12] H. Tran, N.H. Ngo, and J.-M. Hartmann. Efficient computation of some speed-dependent isolated line profiles. J. Quant. Spectrosc. E Radiat. Transfer, 129:199 - 203, 2013. doi: 10.1016/j.jqsrt.2013.06.015. Erratum: JQSRT 134, 104 (2014).

[13] J. Humlíček. Optimized computation of the Voigt and complex probability function. J. Quant. Spectrosc. \& Radiat. Transfer, 27:437-444, 1982. doi: 10.1016/0022-4073(82) 90078-4.

[14] J. Humlíček. An efficient method for evaluation of the complex probability function: the Voigt function and its derivatives. J. Quant. Spectrosc. \& Radiat. Transfer, 21:309-313, 1979. doi: 10.1016/0022-4073(79)90062-1.

[15] J.A.C. Weideman. Computation of the complex error function. SIAM J. Num. Anal., 31:1497-1518, 1994. doi: 10.1137/0731077.

[16] M. Abramowitz and I.A. Stegun. Handbook of Mathematical Functions. National Bureau of Standards, AMS55, New York, 1964.

[17] F.W.J. Olver, D.W. Lozier, R.F. Boisvert, and C.W. Clark, editors. NIST Handbook of Mathematical Functions. Cambridge University Press, New York, NY. Print companion to [18.

[18] DLMF. NIST Digital Library of Mathematical Functions. National Institute of Standards and Technology. URL http://dlmf.nist.gov/ Online companion to [17].

[19] A.K. Hui, B.H. Armstrong, and A.A. Wray. Rapid computation of the Voigt and complex error functions. J. Quant. Spectrosc. E Radiat. Transfer, 19:509-516, 1978. doi: 10.1016/ 
0022-4073(78)90019-5.

[20] F. Schreier. Optimized implementations of rational approximations for the Voigt and complex error function. J. Quant. Spectrosc. \& Radiat. Transfer, 112(6):1010-1025, 2011. doi: $10.1016 /$ j.jqsrt.2010.12.010.

[21] F. Rohart, G. Wlodarczak, J.-M. Colmont, G. Cazzoli, L. Dore, and C. Puzzarini. Galatry versus speed-dependent Voigt profiles for millimeter lines of $\mathrm{O}_{3}$ in collision with $\mathrm{N}_{2}$ and $\mathrm{O}_{2}$. J. Mol. Spectrosc., 251(1-2):282 - 292, 2008. doi: 10.1016/j.jms.2008.03.005. Special issue dedicated to the pioneering work of Drs. Edward A. Cohen and Herbert M. Pickett on spectroscopy relevant to the Earth's atmosphere and astrophysics.

[22] G.P.M. Poppe and C.M.J. Wijers. More efficient computation of the complex error function. ACM Trans. Math. Soft., 16:38-46, 1990. doi: 10.1145/77626.77629.

[23] G.P.M. Poppe and C.M.J. Wijers. Algorithm 680 - evaluation of the complex error function. ACM Trans. Math. Soft., 16:47, 1990. doi: 10.1145/77626.77630.

[24] Mofreh R. Zaghloul and Ahmed N. Ali. Algorithm 916: Computing the Faddeyeva and Voigt functions. ACM Trans. Math. Soft., 38(2):15:1-15:22, January 2011. doi: $10.1145 / 2049673.2049679$.

[25] F. Schreier. The Voigt and complex error function: A comparison of computational methods. J. Quant. Spectrosc. \& Radiat. Transfer, 48:743-762, 1992. doi: 10.1016/ 0022-4073(92)90139-U.

[26] S.A. Clough and F.X. Kneizys. Convolution algorithm for the Lorentz function. Appl. Opt., 18:2329-2333, 1979. doi: 10.1364/AO.18.002329.

[27] D.P. Edwards. Atmospheric transmittance and radiance calculations using line-by-line computer models. In Modelling of the Atmosphere, volume 928, pages 94-116. Proc. SPIE, 1988

[28] L. Sparks. Efficient line-by-line calculation of absorption coefficients to high numerical accuracy. J. Quant. Spectrosc. \& Radiat. Transfer, 57:631-650, 1997. doi: 10.1016/ S0022-4073(96)00154-9.

[29] F. Schreier. Optimized evaluation of a large sum of functions using a three-grid approach. Comp. Phys. Comm., 174:783-802, 2006. doi: 10.1016/j.cpc.2005.12.015.

[30] A. Berk, P. Conforti, and F. Hawes. An accelerated line-by-line option for MODTRAN combining on-the-fly generation of line center absorption within $0.1 \mathrm{~cm}^{-1}$ bins and precomputed line tails. volume 9472, pages 947217 - 947217-11, 2015. doi: 10.1117/12. 2177444 . 